\title{
Neck Lymph Node Boundary Sublevel IA
}

National Cancer Institute

\section{Source}

National Cancer Institute. Neck Lymph Node Boundary Sublevel IA. NCI Thesaurus. Code C132495.

The following anatomical structures define the neck lymph node boundary for sublevel IA: Superior: symphysis of the mandible; inferior: body of the hyoid; anterior (medial): anterior belly of the contralateral digastric muscle; posterior (lateral): anterior belly of the ipsilateral digastric muscle. (AJCC 8th ed.) 